DOI: $10.47460 /$ uct.v24i107.416

\title{
VARIACIÓN DEL INDICADOR RIESGO-PAÍS EN EL FLUJO DE INVERSIÓN EXTRANJERA DEL ECUADOR
}

\author{
Calahorrano, Lucía ${ }^{1}$, Tigse, Silvia ${ }^{2}$ y Caicedo, Francisco ${ }^{3}$ \\ $\left\{\right.$ lecalahorrano $^{1}$, sntigse $^{2}$, fmcaicedo $\left.^{3}\right\} @$ espe.edu.ec \\ https://orcid.org/0000-0001-9261-05671, https://orcid.org/0000-0001-7742-0773², \\ https://orcid.org/0000-0002-1065-78963 \\ Universidad de las Fuerzas Armadas ESPE \\ Sangolquí-Ecuador
}

Recibido (09/10/20), Aceptado (28/11/20)

\begin{abstract}
Resumen: La economía ecuatoriana a lo largo de los años ha atravesado un escenario negativo de eventos y acontecimientos que han originado el ascenso del riesgo-país. El objetivo de esta investigación es demostrar el impacto que genera el índice riesgo país en los flujos de inversión directa de la balanza de pagos del Ecuador. El diseño metodológico evidenció un enfoque cuantitativo-predictivo de alcance correlacional, puesto que, se analizó una base de datos con información recabada del Banco Central del Ecuador mediante el software estadístico R-Studio. Se evidenciaron resultados que indican la reducción de inversión extranjera frente a eventuales incrementos en los puntos básicos del índice riesgo-país. En conclusión, la inversión extranjera directa se muestra con una alta sensibilidad ante los eventuales sucesos políticos, macroeconómicos y de inestabilidad social que provoca la alta calificación en los puntos básicos del indicador.
\end{abstract}

Palabras Clave: Inversión extranjera, riesgo-país, balanza de pagos.

\section{VARIATION OF THE COUNTRY RISK INDICATOR IN ECUADOR'S FOREIGN INVESTMENT FLOW}

\begin{abstract}
The Ecuadorian economy over the years has gone through a negative scenario of events and events that have caused the rise in country risk. The objective of this research is to demonstrate the impact that the country risk index generates on direct investment flows in Ecuador's balance of payments. The methodological design evidenced a quantitative-predictive approach of correlational scope, since a database with information collected from the Central Bank of Ecuador was analyzed using the statistical software R-Studio. Results were evidenced that indicate the reduction of foreign investment in the face of eventual increases in the basic points of the country risk index. In conclusion, foreign direct investment is highly sensitive to eventual political, macroeconomic and social instability events caused by the high rating in the basic points of the indicator.
\end{abstract}

Keywords: Foreign investment, country-risk, balance of payments. 


\section{I.INTRODUCCIÓN}

Los ingresos que percibe la balanza de pagos en Ecuador están conformados por inversión extranjera directa, indirecta o de cartera, activos en reservas y otras inversiones [1]. Se considera únicamente a los flujos de capital extranjero directo, para el presente estudio. El objetivo de la investigación, es demostrar el impacto que genera el índice riesgo-país en los flujos de inversión extranjera directa, mediante bases teóricas y la práctica de un modelo econométrico que represente una fuente de información verídica para políticas futuras de comercio exterior, que impulsen la atracción de recursos externos.

En los últimos años uno de los mecanismos que aporta al desarrollo de los países es la inversión extranjera directa [2] denominado de aquí en adelante como IED ya que ha llegado a ser primordial en la integración económica internacional, donde se busca dinamizar la economía de los países a través de la obtención de recursos y tecnologías [3]. La IED se ha transformado en una pieza clave como fuente de financiación para las economías por el efecto que tiene a largo plazo, y la manera en que contribuye en los sectores productivos [4]. Es así, que los inversionistas dentro de su estudio toman la decisión de invertir en cuenta al riesgo-país ya que es uno de los determinantes en los flujos de inversión [5]. Ecuador se ha visto afectado en relación al comportamiento irregular en los flujos de capital extranjero, por diversas circunstancias macroeconómicas, políticas y de inestabilidad social, que han sido la raíz de una alta calificación en el indicador riesgo-país.

Partiendo de los diferentes montos y datos establecidos durante el I trimestre del año 2010 al I trimestre del año 2020, es preciso analizar la variación del indicador riego-país y los flujos de inversión extranjera directa, cuya validez se encuentra evidenciado en el modelo econométrico simulado en el software estadístico R-Studio. Por esta razón, se formula la hipótesis de investigación, la cual establece que el indicador riego-país influye en los flujos de IED y así se genera cierta incertidumbre en los inversionistas en el momento de la toma de decisiones para el aporte de sus capitales en la economía ecuatoriana.

La presente investigación se organiza de la siguiente manera: resumen, como sección I la introducción, sección II el desarrollo de la investigación que presenta los términos teóricos y relación entre el riego-país y la IED, seguido de la sección III la metodología que se expuso en la investigación, posteriormente la sección IV los resultados establecidos y finalmente las conclusiones a las que se llegó y referencias en las que se sustentaron la investigación.

\section{II.DESARROLLO}

Los ingresos que recibe el Ecuador en la cuenta financiera, correspondiente a la balanza de pagos en la que trata de la variación de los activos y pasivos de la economía local frente al resto del mundo, se conforma por: inversión directa, que refleja el interés duradero de un inversionista directo en una entidad residente de otra economía y abarca todas las transacciones realizadas entre ellos, así como la gestión e influencia considerable del inversionista en la dirección de la firma; la segunda, es la inversión de cartera que corresponde a las transacciones de títulos de participación en el capital y títulos de deuda, seguido por otras inversiones que tratan el registro de las transacciones financieras como créditos comerciales, prestamos, depósitos y otros activos y pasivos otorgados por agentes del exterior a residentes ecuatorianos, $\mathrm{y}$, por último los activos en reserva donde se incluye todas las transacciones que las autoridades monetarias de un estado consideran disponibles para atender necesidades de financiamiento [6].

Es fundamental reconocer cuales son aquellos componentes que exponen a la IED y como incide el riego-país en sus flujos.

\section{A.Inversión Extranjera Directa}

Es aquella aportación por parte externa al país, ya sea de personas naturales o jurídicas [7]. Su importancia reside en el ingreso de capitales frescos al estado para estimular la economía nacional, optimizando la productividad y lograr mayor competitividad [8].

Los beneficios que abarca hacen que los diferentes gobiernos tiendan a instituir políticas para captar un número mayor de inversiones que incentiven el crecimiento económico y minimizar el desempleo, de igual manera cumple un rol de suma importancia en el financiamiento de países en vías de desarrollo [9].

La orientación de los diferentes flujos de IED se ven determinados por variables que influyen directamente en la decisión del inversionista como son apertura comercial estabilidad macroeconómica, tamaño de mercado, políticas comerciales, solvencia económica del país receptor [10].

\section{B.Evolución de la IED}

El Ecuador, históricamente se ha beneficiado de manera mínima de la inversión extranjera debido a una serie de reformas en materia económica y fiscal, así, siendo uno de los países con menor captación de IED [11], a su vez un determinado número de empresarios exigen otorgar incentivos para atraer capitales extranjeros, contrarrestar el desempleo y dinamizar el crecimiento de la producción en el país [12]. 
Los flujos que se han captado en el periodo enero del año 2000 a marzo del año 2020 indican un comportamiento irregular, además los principales sectores a los que se destinó son explotación de minas y canteras, manufactura, comercio, servicios prestados a las empresas y agricultura [13]. Entre los países con mayor peso de IED se tiene a: México, Holanda, Canadá, China y España.

Entre los años con mayor enfoque en IED, el año 2008 en el que la economía ecuatoriana empezó a ganar estabilidad, y existió una transformación lo cual hizo que capitales extranjeros ingresen al país con expectativa en el gobierno entrante del Econ. Rafael Correa donde alcanzó una cifra récord de USD 1056,7 millones [14].
En el año 2015, de acuerdo con la información estadística proporcionada por la Comisión Económica para América Latina y el Caribe [15], la IED alcanzó los USD 1322,6 millones, dado que se incorporó proyectos por medio de concesiones y contratos. Para el año 2016, disminuye en cuanto a razones que provocaron el descenso de capital considerando una de ellas, al terremoto que tuvo lugar en abril llegando a USD 764,40 millones, y a partir de ahí, se evidenciaron flujos de capital externo en el Ecuador que variaban constantemente, y ya, en el año 2018 presentó un ascenso por motivo a la vigencia de la Ley de Fomento Productivo provocando de esta manera una estabilidad política y tributaria, obteniendo una cifra histórica de USD 1455,70 millones [16] (Ver figura 1).

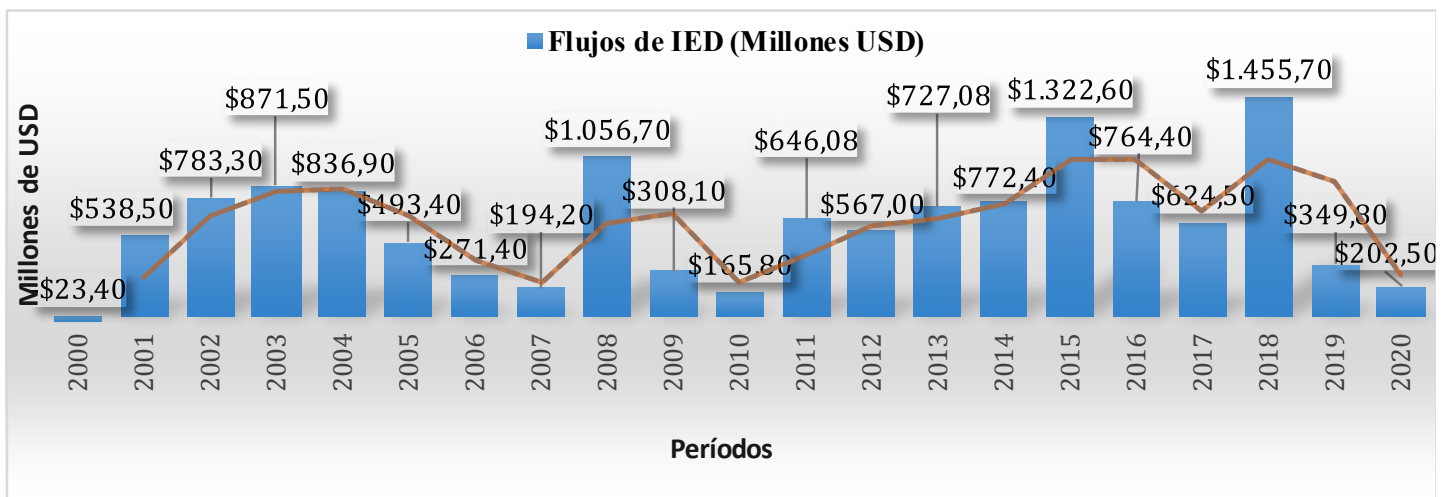

Figura 1. Evolución de la inversión extranjera directa desde el período enero del año 2000 a marzo del año 2020

Fuente: Banco Central del Ecuador

\section{C.Riego-país}

Mide la capacidad de un estado para cumplir con los pagos de los intereses y del principal de un crédito al momento de su vencimiento, determina la predisposición de un estado al hacer frente a sus obligaciones y está orientado a proteger la rentabilidad de los inversionistas [16].

El papel del riego-país es crucial en un nivel institucional en cada uno de los países ya que depende de las inversiones para crecer y desarrollarse de manera global, y que sólo llegarán al estado en la medida en que el indicador sea bajo; cuanto menor sea, mayor será la entrada de inversiones, los diferentes factores políticos, sociales, macroeconómicos y naturales son variables determinantes ya que generan una variación en la toma de decisiones [17], de esta manera, es un indicador que ha logrado formar parte del lenguaje cotidiano en las principales economías de América Latina [18].

\section{D.Evolución del riego-país}

En Ecuador, según el historial del riego-país, en el año 2000 oscilaba los 3926 puntos, debido a la crisis financiera en medio de la dolarización [20], para el año 2008 se evidencia un dramático salto a los 4731 puntos, debido al anuncio del gobierno de no cancelar la deuda externa declarándola como ilegitima, además de la caída del precio del petróleo en menos de USD 35,00 [19], posterior a este suceso se observa al indicador en el año 2020 los 4553 puntos dado que la Asamblea Nacional sugiere no pagar la deuda y utilizar los recursos en la emergencia sanitaria por el covid-19 lo que generó incertidumbre en los mercados internacionales, a pesar que esto se terminó pagando (Ver figura 2). 


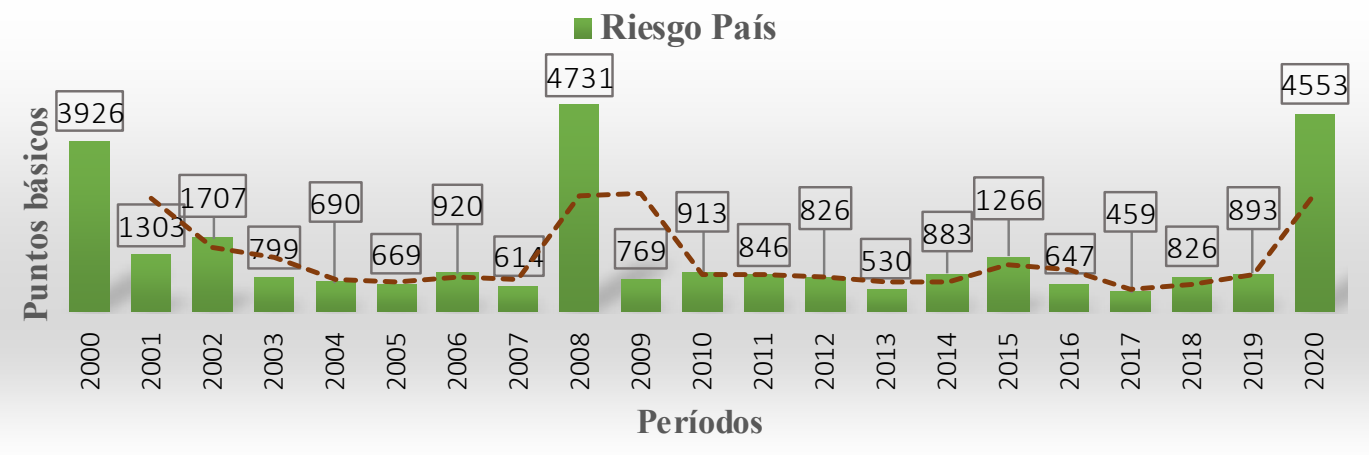

Figura 2. Evolución del indicador Riego-país desde el período enero del año 2000 a marzo del año 2020 Fuente: Banco Central del Ecuador

\section{III.METODOLOGÍA}

El presente estudio se desarrolló a partir de una metodología de enfoque cuantitativo, bajo el método deductivo-predictivo. Así, el diseño del modelo se basó en datos históricos, analizados desde el I trimestre del año 2010 al I trimestre del año 2020, serie de tiempo compuesta por 41 observaciones y en la que cabe destacar que cumple con la característica de periodicidad al momento de emplear métodos econométricos para el análi- sis de variables de orden [20]. En cuanto a la modalidad utilizada, fue bibliográfica, recolectada del boletín de la balanza de pagos, e información estadística mensual emitidos por el Banco Central del Ecuador [21].

La Tabla I refleja la muestra de los flujos de inversión extranjera directa que registró la cuenta financiera de la balanza de pagos ecuatoriana y la evolución en puntos básicos del indicador riego-país, a partir de los últimos diez años y el primer trimestre del año 2020.

TABLA I. Flujos de inversión extranjera y puntos básicos del riego-país

\begin{tabular}{|c|c|c|c|c|c|}
\hline Período & $\begin{array}{c}\text { Inversión } \\
\text { Extranjera Directa } \\
\text { (millón USD) }\end{array}$ & Riego-país & Período & $\begin{array}{c}\text { Inversión } \\
\text { Extranjera Directa } \\
\text { (millón USD) }\end{array}$ & Riego-país) \\
\hline & 2010 & & & 2015 & \\
\hline Marzo & $-174.892 .280,00$ & 817 & Marzo & $183.235 .516,00$ & 865 \\
\hline Junio & $212.272 .479,00$ & 1013 & Junio & $96.828 .476,00$ & 824 \\
\hline Septiembre & $159.377 .176,00$ & 1226 & Septiembre & $259.440 .756,00$ & 1451 \\
\hline Diciembre & $\begin{array}{l}-30.891 .055,00 \\
\mathbf{2 0 1 1}\end{array}$ & 913 & Diciembre & $\begin{array}{l}783.196 .589,00 \\
\mathbf{2 0 1 6}\end{array}$ & 1266 \\
\hline Marzo & $193.353 .834,00$ & 780 & Marzo & $198.611 .931,00$ & 1058 \\
\hline Junio & $159.816 .813,00$ & 783 & Junio & $128.867 .849,00$ & 913 \\
\hline Septiembre & $67.355 .543,00$ & 868 & Septiembre & $129.544 .980,00$ & 845 \\
\hline Diciembre & $\begin{array}{l}225.551 .169,00 \\
\quad \mathbf{2 0 1 2}\end{array}$ & 846 & Diciembre & $\begin{array}{l}311.972 .108,00 \\
\mathbf{2 0 1 7}\end{array}$ & 647 \\
\hline Marzo & $109.518 .820,00$ & 824 & Marzo & $184.780 .156,00$ & 666 \\
\hline Junio & $181.204 .076,00$ & 892 & Junio & $188.850 .438,00$ & 706 \\
\hline Septiembre & $86.052 .882,00$ & 743 & Septiembre & $148.876 .240,00$ & 606 \\
\hline Diciembre & $\begin{array}{l}190.634 .523,00 \\
\quad \mathbf{2 0 1 3}\end{array}$ & 826 & Diciembre & $\begin{array}{l}96.338 .578,00 \\
\mathbf{2 0 1 8}\end{array}$ & 459 \\
\hline Marzo & $113.934 .791,00$ & 700 & Marzo & $331.519 .767,00$ & 544 \\
\hline Junio & $281.996 .456,00$ & 665 & Junio & $208.918 .857,00$ & 761 \\
\hline Septiembre & $115.863 .681,00$ & 628 & Septiembre & $260.298 .432,00$ & 621 \\
\hline Diciembre & $\begin{array}{l}215.269 .279,00 \\
\mathbf{2 0 1 4}\end{array}$ & 530 & Diciembre & $\begin{array}{l}609.698 .090,00 \\
\mathbf{2 0 1 9}\end{array}$ & 826 \\
\hline Marzo & $136.845 .997,00$ & 508 & Marzo & $209.602 .643,00$ & 592 \\
\hline Junio & $160.862 .156,00$ & 376 & Junio & $280.596 .538,00$ & 580 \\
\hline Septiembre & $145.066 .009,00$ & 401 & Septiembre & $128.635 .482,00$ & 677 \\
\hline \multirow[t]{2}{*}{ Diciembre } & $329.615 .784,00$ & 883 & Diciembre & $\begin{array}{l}318.789 .607,00 \\
\quad \mathbf{2 0 2 0}\end{array}$ & 826 \\
\hline & & & Marzo & $202.501 .839,00$ & 4553 \\
\hline
\end{tabular}

Fuente: Banco Central del Ecuador (BCE) 
El nivel de la investigación permitió examinar la interrelación simultánea, entre las respectivas variables cuantitativas, como son: flujos de inversión extranjera directa y el indicador riego-país, de esta manera se pretendieron alcanzar el objetivo y la comprobación de las hipótesis, tras la especificación y enfoque de la modelación econométrica causal o modelo de vectores autorregresivos (VAR). El cual consiste en determinar si a través del tiempo existe una relación de causa y efecto, sobre las variables económicas ante un determinado shock [22]. La aplicación del modelo de vectores autorregresivos (VAR), se concretó en el software estadístico R-Studio herramienta de entorno interactivo, calificado como un sistema poderoso en lenguaje de programación, sofisticada capacidad de gráficos y funcionalidad de paquetes modulares, diseñado para ser aplicado en el procesamiento de datos en ciencias [23], el mismo en donde se desarrolló la estimación de las ecuaciones autorregresivas en función de rezagos, la verificación del modelo por medio de un diagnóstico de especificación en los residuales, el cual fue: auto correlación serial, normalidad, heteroscedasticidad o varianza constante y estabilidad, seguido de la prueba de causalidad en el sentido de Granger, función impulso respuesta y descomposición de la varianza.

\section{IV.RESULTADOS}

La investigación partió de la especificación del modelo de vectores autorregresivos VAR, tomando en consideración una serie de datos de periodicidad trimestral, en donde se corroboró el impacto causal de la variación del indicador riego-país sobre los ingresos de inversión extranjera directa. Previo la estimación y calibrado econométrico VAR, se aplicó la prueba de raíz unitaria de Dickey-Fuller Aumentada (ADF) dando como resultados un test-statistic de $-7,75$ y $-2,61$ puntos significativos sobre los resultados en los porcentajes de un $1 \%$ - $5 \%-10 \%$ y $5 \%-10 \%$ en las variables IED y riego-país de forma respectiva, de esta manera se concluye que la serie de datos satisface el supuesto de ruido blanco, que, bajo el marco econométrico es importante verificar la estacionariedad de los datos para obtener mejores resultados a la hora de estimar y pronosticar [24] (ver figura 3). Por lo tanto, se procedió a la selección de los rezagos óptimos, en donde se utilizó los criterios de información AIC, HQ, SC y FPE, y se consiguió como resultado un número de 12 rezagos.

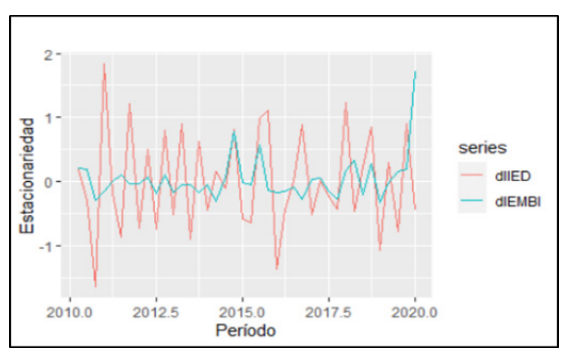

Figura 3. Prueba de Dickey-Fuller Aumentada y diagrama de estacionalidad

Fuente: Software estadístico R-Studio

Una vez detectado el regazo óptimo para el modelo VAR se realizó la estimación, lo que determinó las ecuaciones autorregresivas de ambas variables, dentro de los resultados, se visualizó las raíces del polinomio característico, en donde indicó un error de especificación en una mínima expresión al presentar polinomios que sobrepasan el círculo de la unidad, así también tuvo operadores menores a uno, que resaltó la estabilidad de los residuos que se comprobó con las pruebas de especificación que se realizó al modelo para conocer las dificultades. Como se evidenciaron en los resultados arrojados por el software, señaló que la variable endógena IED tiene un impacto significativo en su valor actual determinado por rezagos de su propia expresión conjuntamente con los de la variable riego-país, además de una matriz de covarianza y correlación que indicó el efecto inverso que existe, lo que quiere decir que los flujos de capital extranjero tienden a decrecer ante un impacto significativo en el índice riego-país. Este modelo estuvo referenciado por los p-valor de 0,0285 en IED y 0,6728 en el indicador riego-país, siendo así, que se consideró la ecuación estimada de la variable IED para responder la hipótesis de investigación, al presentar valores estadísticos significativos como un $\mathrm{R}^{\wedge} 2$ de $99 \%$ de la bondad de ajuste, que representa el efecto global de una variable sobre otra y cierta significancia en los retardos que conforman la ecuación. A continuación, se detalla los resultados: 


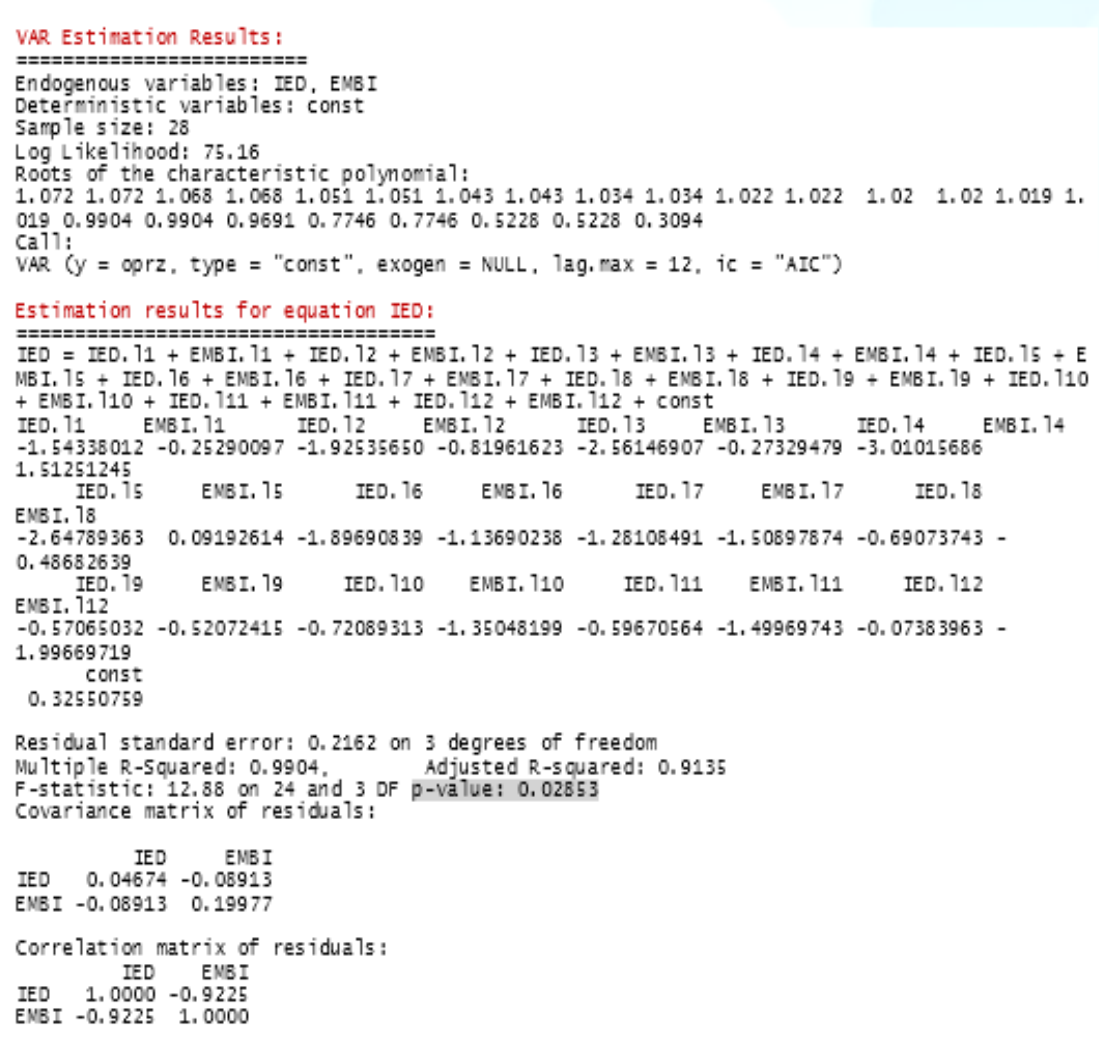

Figura 4: Estimación del modelo VAR

Fuente: Software estadístico R-Studio.

Después de haber realizado la estimación del modelo VAR se verificó que cumpla con la condición de estabilidad en los residuos, en donde el resultado de test de auto correlación indicó un p-valor de 2,2e-16 que concluyó la existencia de correlación serial, en cuanto a la prueba de normalidad, mostró ausencia de distribución normal en el sesgo con un p-valor de 0,01447 representativo en una mínima expresión por la presencia de valores externos en los errores [25], no presentó problemas en la curtosis y en la prueba estadística de Jarque-Bera, dando como resultados un p-valor de 0,3824 y 0,03429 de manera respectiva, seguido por la prueba de heroscedásticidad de ARCH donde se comprobó un test-statistic de 0,0799 aceptando la hipótesis nula y se determinó que los errores son homoscedásticos. Finalmente, a través de las respuestas evidentes en las pruebas de estabilidad se detectó una rotura estructural en el año 2010 debido a la caída en los ingresos de inversión directa, provocando desventajas con el supuesto de normalidad y generando un impacto negativo en los posibles pronósticos justificados en variaciones marcadas por antecedentes de los períodos analizados (Ver figura 5).

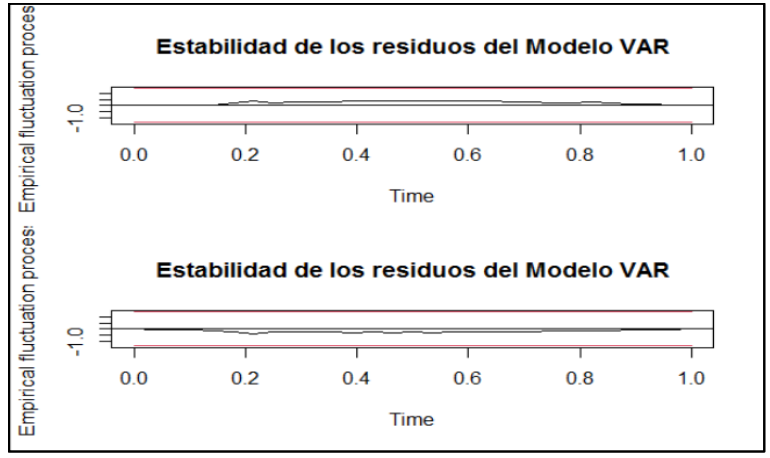

Figura 5. Diagrama de estabilidad de los residuales.

Además, se realizó un test de causalidad en el sentido de Granger, en donde se comprobó con un p-valor menor a 0,05 en un orden de integración 1 , se aceptó la hipótesis alternativa de la prueba causal la cual reafirma la existencia de causalidad entre la variación del indicador riego-país en el ingreso de inversión extranjera directa.

Con el propósito de evidenciar la interacción dinámica del modelo se concretó el análisis de la función impulso-respuesta, herramienta que consiste en la simulación de los efectos de shock en las distintas perturbaciones aleatorias y posteriormente la descomposición de la varianza del error de predicción para detectar los 
factores asociados a cada perturbación delimitada [26] [27]; modalidades que pueden darse por los diversos aspectos que engloban la calificación del indicador riego-país dentro de la inversión directa.

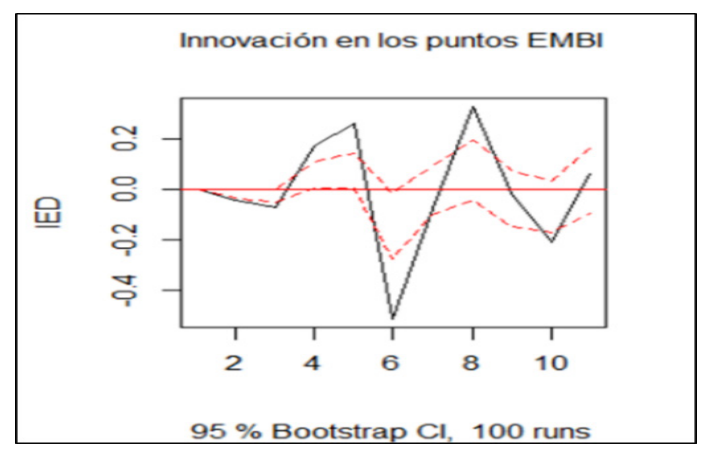

Figura 6. Impulso- respuesta de las variables IED

Con la estimación del modelo para los flujos de inversión extranjera directa, se utilizó la función impulso-respuesta para realizar el análisis del comportamiento que tendrá a escenarios futuros ante la innovación en los puntos básicos del indicador riego-país, provocando un efecto negativo con porcentajes del $-4,36 \%,-7,03 \%$, $-51,7 \%,-7,65 \%-1,68 \%$ y un $-20,89 \%$ en los años 2 , $3,6,7,9$ y 10 respectivamente. De esta manera, se establece que el comportamiento a futuro del ingreso de capitales extranjeros puede estar ligado a eventos que el país sobrellevo en el pasado. Con los resultados expuestos en la Figura 6 se pronosticó la reacción en los inversionistas directos en el trascurso de 10 años, la cual puede deberse por diversos acontecimientos subjetivos que pueda percibir la economía ecuatoriana, en donde sean los causantes del impacto en puntos volátiles del indicador y la consecuencia de la caída de flujos por inversiones directas, entre ellos se resalta: índices de inflación creciente, déficit en la balanza de pagos, sobreendeudamiento externo, reformas de ley, barreras arancelarias, paralización de actividades, incapacidad de repatriar capitales, dividendos, corrupción, dependencia de ingresos petroleros y desestabilización sociopolítica, modalidades económicas, políticas y sociales que se han visto al largo de los años, perjudicar la imagen del país en el mercado internacional y exponiéndolo como insolvente y contrayente de una baja tendencia en la competitividad comercial en el mercado exterior.

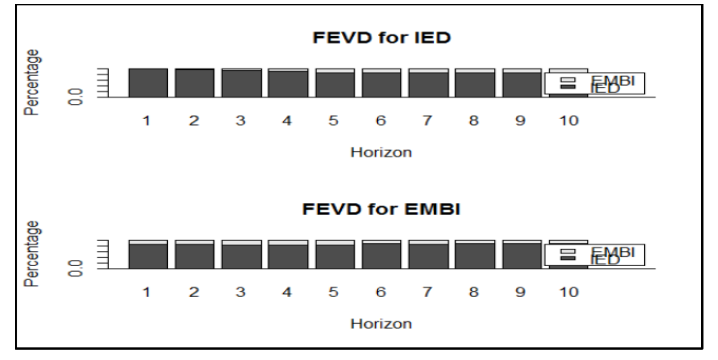

Figura 7. Descomposición de la varianza de las variables.

La descomposición de la varianza del modelo autorregresivo, indicó el porcentaje de volatilidad que registra cada una de las variables exógenas, en donde los porcentajes de la variable inversión extranjera directa se muestra propensa a disminuir a través del tiempo, caso contrario se muestra en la reacción de volatilidad del indicador riego-país, que obtiene porcentajes estables para las perturbaciones aleatorias que se mantiene a valor futuro.

\section{V.CONCLUSIONES}

El Ecuador a través de los años no ha sido bien visto por los inversionistas extranjeros por la inestabilidad política, desastres naturales, en los que se generó incertidumbre tanto nacional como internacional y los montos de IED decrecieron. Así mismo, los puntos de riego-país se han incrementado, por lo que la cantidad de dinero que ha ingresado al país ha disminuido, a pesar de esto en el año 2015 se logró incorporar varios proyectos de inversión extranjera, por medio de contratos enfocados principalmente en el sector petrolero y en la construcción de infraestructura del país, el riego-país se ve en ventaja debido a la implementación de diferentes reformas financieras, monetarias, laborales y lo más trascendental fue el acuerdo con la Unión Europea firmado el 16 de julio del 2014 lo que representa escenarios favorables para la inversión.

Con los resultados alcanzados en el presente análisis de modelación econométrica de vectores autorregresivos, se ratificó el impacto significativo en los flujos de la inversión extranjera directa de la balanza de pagos por el creciente índice de riesgo-país, debido a las distintas situaciones y eventos que ocasiona su constante crecimiento, a medida que refleja circunstancias relevantes de acontecimientos percibidos bajo un marco macroeconómico, político y social, en donde los más significativos en relación a la inversión directa, son: riesgos de incumplimiento de compromisos como dividendos, sobreendeudamiento externo, desestabilización sociopolítica, barreras arancelarias, escasas políticas de 
comercio exterior y restricciones a la salida de divisas. Situaciones que a largo de los años han sido la consecuencia de un bajo desarrollo sostenible en los ingresos canalizados del mercado internacional, y que, en ocasiones, la decisión de los inversionistas para el cierre de actividades de empresas transnacionales en el país, y destinando los capitales a entornos que conlleven beneficios a un largo plazo que se concreten en proyectos de productividad, competitividad y empleo para ambos lados.

Ecuador es un país competitivo con una economía dinámica, ubicación geográfica privilegiada, sin embargo, en los últimos 40 años ha registrado el mismo problema que es no atraer capitales, recibiendo un $0,76 \%$ de la inversión total que llega a América Latina, lo que significa que el estado debe mejorar significativamente las cifras en este rubro, por ello es fundamental que se recupere la confianza en el inversionista, creando estabilidad política y jurídica dejando de lado las limitaciones con barreras que ahuyenta la atracción de capitales extranjeros. Es por eso, que a partir del año 2018 entra en vigencia la Ley de Fomento Productivo lo que provocó estabilidad política y tributaria, consiguiendo ser uno de los años con mayor atracción en inversiones y generando mayores expectativas para el futuro.

\section{REFERENCIAS}

[1]Banco Central del Ecuador, «Notas metodológicas de la balanza de pagos del Ecuador,» BCE, Quito, 2018. [2]J. Armijos, «Efecto de la inversión extranjera directa en el crecimiento económico en Ecuador,» Revista Económica, vol. 2, nº 1, pp. 31-38, 2017.

[3]J. López and J. Laverde, «Inversión Extranjera Directa y carga tributaria en Ecuador, periodo 20042016,» Valor Agregado, vol. 5, no 7, pp. 50-53, 2017. [4]G. Rodriguez and D. Forero, «Factores determinantes de la inversión extranjera directa,,» Universidad Tecnológica de Bolivar, vol. 2, pp. 119-134, 2016.

[5]S. Cárdenas and J. Behr, «La inversión extranjera y el riesgo país en el Ecuador, Periodo 2007-2013,» Revista Observatorio de la Economía Latinoamericana, Ecuador, 2016.

[6]Banco Central del Ecuador, «Análisis trimestral de la balanza de pagos,» BCE, Quito, 2019.

[7]D. Rosillo, Análisis de la inversión extranjera directa en el ecuador, la afectación del índice riesgo país en la captación de inversiones y su incidencia en la economía de las empresas en el período 2007-2010, Quito, 2011. [8]A. Espín and A. Villalva, Análisis de la inversión extranjera directa y sus determinantes en el Ecuador, período 2007-2015, Quito, 2017.

[9]O. Cedeño and Á. Mendoza, «Impacto del índice riesgo país en la inversión extranjera directa de Ecuador periodo 2016-2018,» Polo de Conocimiento, vol. 5, $\mathrm{n}^{\circ}$ 03, pp. 619-639, 2020.

[10]J. Mogrovejo, «Factores determinantes de la inversión extranjera directa en algunos países de Latinoamérica,» Latinoamericana de Desarrollo Económico, vol. 5, pp. 51-82, 2005.

[11]C. Silvia, Impacto del riesgo país en la inversión extranjera directa en el Ecuador en el período 20052015, Cuenca, 2018.

[12]Organización de las Naciones Unidas ONU, «Análisis de las políticas de inversión Ecuador,» de Conferencia de las Naciones Unidas Sobre Comercio y Desarrollo, Nueva York y Ginebra, 2012.

[13]J. Espín, A. Córdova, and G. López r,» Retos- Revista de Ciencias de la Administración y Economia, vol. 6, $\mathrm{n}^{\mathrm{o}} 12$, p. 215, 2016.

[14]J. Soliz, La inversión extranjera directa en el Ecuador y su incidencia en la economía, periodo 2008 2013, Quito, 2015.

[15]E. Zurita, G. González, and E. Borja «Análisis de la dolarización y sus efectos sobre la inversión extranjera directa en Ecuador. Periodo 2000 - 2016,» Bolentín de Coyuntura, vol. 1, p. 4, 2017.

[16]OXFAM, «Ecuador 2016 Respuesta al terremoto,» Ecuador, 2017.

[17]A. Acosta, El otro riesgo país, Quito-Ecuador: Ediciones Abya-Yala, 2005.

[18]L. Domenech and J. Diaz, Análisis de la evolución del riesgo país en la inversión extranjera directa ecuatoriana. período 2003 -2007, Guayaquil, 2008.

[19]J. Erazo and K. Lindao Riesgo Pais Ecuador: "Principales determinantes y su incidencia", Guayaquil, 2004, pp. 1-77, 2004.

[20]C. Larrea, «Crisis, dolarización y pobreza en Ecuador,» Retos para la integración social de los pobres en América Latina, vol. 1, pp. 217-227, 2005.

[21]E. G. Montenegro Cueva, «Análisis de riesgo de las acciones de las empresas más representativas que estructuran el mercado de valores del Ecuador,» Quito, 2014.

[22]J. M. Wooldridge, Introducción a la econometría. Un enfoque moderno, Michigan : South-Western, 2010. [23]Banco Central del Ecuador, «Información estadística monetarias y financieras mensuales,» 31032020. [En línea]. Available: https://contenido.bce.fin.ec/ home1/estadisticas/bolmensual/IEMensual.jsp.. [Último acceso: 1507 2020].

[24]J. Galán, «Equilibrios y conjeturas,» Cuadernos del Seminario de Credibilidad Macroeconómica, vol. 1, p. $1,2014$.

[25]J. S. Santana, «El arte de programar en R,» Instituto 
Mexicano de Tecnologia del Agua, vol. 1, 2014.

[26]L. Mahadeva, «Prueba de raíz unitaria para ayudar a la construcción de un modelo,» Centro de Estudios Monetarios Latinoamericanos, vol. 1, p. 54, 2009.

[27]H. Catalán, «Econometría y análisis de políticas fiscales,» University of Minnesota, vol. 48.

[28]A. Novales, «Modelos vectoriales autoregresivos,» Universidad Complutense, vol. 1, 2017.

[29]T. Calagua, «La Metodología del Vector Autorregresivo : Presentación y Algunas Aplicaciones,» Ciencias Empresariales, vol. 2, pp. 103-108, 2010.

\section{RESUMEN CURRICULAR}

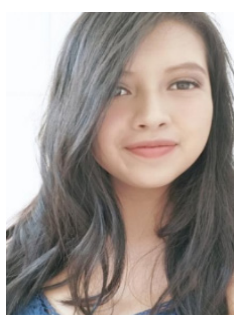

Lucía Elizabeth Calahorrano Yánez, egresada de la carrera de Ingeniería en Finanzas y Auditoría CPA, de nacionalidad ecuatoriana, obtuvo el certificado en calidad de ponente en "Jornada de socialización de proyectos integradores - Generando Productos e Investigación para el fortalecimiento de la gestión del Conocimiento".

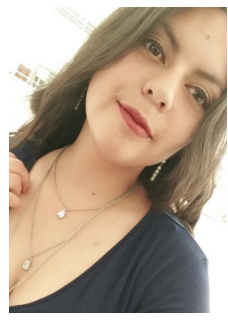

Silvia Nataly Tigse Here dia, egresada de la carrera de Ingeniería en Finanzas y Auditoría CPA, de nacionalidad ecuatoriana, participante en congresos organizados por el Departamento de Ciencias Económicas Administrativas y de Comercio y proyectos integradores de la Universidad de las Fuerzas Armadas ESPE.

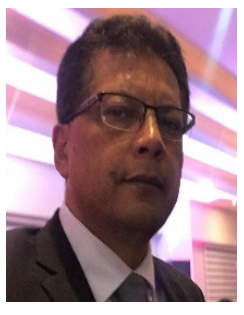

Francisco Marcelo Caicedo Atiaga, Economista por la Universidad Central del Ecuador, Diplomado en Gestión del Aprendizaje Universitario por la Escuela Politécnica del Ejército (Actualidad), Master en Admin istración de Empresas por la Escuela Politécnica del Litoral, Master en Finanzas Corporativas por la Universidad de Viña del Mar Chile, candidato a PHD en Economía y Ciencias Sociales por la Universidad de Carabobo en Venezuela. 